\title{
Determination of Pathophysiological Mechanisms of ALPPS in an Animal Model Using Molecular and Functional Imagings
}

\section{Ruifeng Wang}

Fourth Affiliated Hospital of Harbin Medical University

\section{Zhen Quan}

Fourth Affiliated Hospital of Harbin Medical University

Tongsen Zheng

Tumor Hospital of Harbin Medical University

\section{Kai Wang}

Fourth Affiliated Hospital of Harbin Medical University

Yang Liu

Fourth Affiliated Hospital of Harbin Medical University

\section{Zhaoguo Han}

Fourth Affiliated Hospital of Harbin Medical University

\section{Xiance Wang}

Fourth Affiliated Hospital of Harbin Medical University

\section{Shiling Ma}

Fourth Affiliated Hospital of Harbin Medical University

\section{Lianxin Liu}

USTC: University of Science and Technology of China

\section{Wan Yee Lau}

The Chinese University of Hong Kong

Xilin Sun ( $\nabla$ sunxl@ems.hrbmu.edu.cn )

Fourth Affiliated Hospital of Harbin Medical University

\section{Research}

Keywords: liver cancer, future liver remnant, choline metabolism, proliferation, vascular response

Posted Date: October 8th, 2020

DOI: https://doi.org/10.21203/rs.3.rs-86065/v1 
License: (c) (i) This work is licensed under a Creative Commons Attribution 4.0 International License. Read Full License 


\section{Abstract}

Background: ALPPS (associating liver partition and portal vein ligation for staged hepatectomy) is a twostage strategy to induce rapid hypertrophy of future liver remnant (FLR) to increase hepatic tumor resectability and reduce postoperative liver failure rate. Rigorous and accurate determination of the pathophysiological mechanisms involved in hypertrophy of FLR in ALPPS is essential to ensure a good success rate in the second stage operation.

Methods: An ALPPS model was established in rabbits with liver VX2 tumor. The pathophysiological mechanisms after the first stage of ALPPS in the FLR and tumor were assessed by multiplexed positron emission tomography (PET) tracers and dynamic contrast enhanced magnetic resonance imaging (DCEMRI).

Results: The tumor volume in the ALPPS model increased significantly from post-stage 1 ALPPS day 14 compared to control animals. The ${ }^{18}$ F-FDG uptake increased significantly from day 7 onwards in the ALPPS model. A Valid Volumetric Function measured by ${ }^{18} \mathrm{~F}-\mathrm{FCH}$ showed good values in accurately monitoring dynamics and time window for functional liver regeneration (days 3 to 7). DCE-MRI revealed changes in the vascular hyperpermeability function, with a peak on day 7 for tumor and FLR.

Conclusions: Molecular and functional imagings are promising and non-invasive methods to investigate the pathophysiological mechanisms of ALPPS with good potentials in clinical application to improve surgical successful outcomes.

\section{Introduction}

Liver cancer is a common malignant tumor globally [1]. Although many treatments are currently available, surgical resection remains the main treatment aiming at cure for liver cancer patients with preserved liver function [2]. The volume and functional reserve of future liver remnant (FLR) are critical for clinical outcomes after extensive liver resection, as post-hepatectomy liver failure (PHLF) is an important cause of morbidity and mortality [3]. While portal vein ligation (PVL) or portal vein embolization (PVE) can be used to induce hypertrophy of FLR, these methods require long waiting intervals for adequate liver hypertrophy in FLR for the second stage operation, thus carrying a significant risk of tumor progression leading to unresectability during the wait [4]. Schnitzbauer et al first reported the novel two-stage hepatectomy procedure which was named later as "Associating Liver Partition and Portal vein ligation for Staged hepatectomy (or ALPPS in short)" [5]. The first stage of ALPPS involves portal vein ligation and in situ splitting of liver parenchyma, whereas the second stage consists of completion hepatectomy after adequate hypertrophy of FLR. ALPPS results in more rapid liver hypertrophy than PVE or PVL, thus it can provide an opportunity for patients with primarily unresectable liver tumors to undergo curative resection [4].

Liver function and tumor behavior are the two most important factors influencing the timing of the second stage of ALPPS and the outcomes of treatment. However, the pathophysiological mechanisms 
after the first stage of ALPPS are poorly understood. Although previous clinical studies have utilized measurement of FLR volume by computed tomography (CT) as the standard to select patients for ALPPS, volume changes do not necessarily reflect hepatic function [6]. Moreover, whether the first stage of ALPPS promotes tumor progression remains debatable. In addition, it is necessary to investigate the correlation between liver function of the FLR and tumor behavior which primarily determine the clinical outcomes of ALPPS $[7,8]$.

Molecular and functional imagings are valuable in diagnosing liver diseases, studying non-invasively pathophysiological molecular mechanisms, and evaluating therapeutic responses. Positron emission tomography (PET), when combined with CT scan, provide both anatomical and molecular information. Furthermore, multiparametric dynamic contrast-enhanced magnetic resonance imaging (DCE-MRI) can quantitatively reflect vascular permeability of tissue angiogenesis $[9,10]$.

Among the PET radiotracers used in clinical research, glucose analog 2-deoxy-2- ${ }^{18} \mathrm{~F}$-fluoro-D-glucose $\left({ }^{18} \mathrm{~F}\right.$ FDG) is widely used to detect a variety of cancers based on the accelerated rates of glycolysis in tumor

cells. ${ }^{18} \mathrm{~F}$-fluorothymidine $\left({ }^{18} \mathrm{~F}\right.$-FLT) has also been used as it accumulates in proliferating cells by binding to thymidine kinase 1 (TK-1). Moreover, as the liver is a central organ for choline metabolism and as hepatic dysfunction causes important changes in phospholipid metabolism, the ${ }^{18} \mathrm{~F}$-labeled choline analog, ${ }^{18} \mathrm{~F}$-fluorocholine $\left({ }^{18} \mathrm{~F}-\mathrm{FCH}\right)$, has also been used as a PET biomarker to detect various liver diseases [11-15].

In this study, an ALPPS model was established in rabbits with VX2 liver tumor. The pathophysiologic mechanisms of ALPPS were investigated by monitoring volume changes, choline and glucose metabolisms, proliferation, and vascular responses in FLR and tumor with multimodal molecular and functional technologies. This study lays novel empirical groundworks in designing effective treatment strategies for liver diseases (Fig. 1).

\section{Materials And Methods}

\section{Experimental Design}

Two weeks after VX2 tumor implantation, the tumor-bearing rabbits were randomly divided into the ALPPS group (with the left branch of portal vein being ligated and the liver parenchyma being split between the left medial lobe and right lobe) and the Sham group (with the hepatic artery and portal vein being dissected without ligated). Three rabbits were selected from each group to have a DCE-MRI scan and a PET/CT scan with ${ }^{18} \mathrm{~F}-\mathrm{FDG},{ }^{18} \mathrm{~F}-\mathrm{FCH}$, and ${ }^{18} \mathrm{~F}-\mathrm{FLT}$ on days $0,1,3,7$ and 14 after surgery. After imagings, the rabbits were sacrificed for future pathological studies.

\section{The ALPPS model in rabbits with liver VX2 tumor}

This study was approved by the Animal Care Committee of Harbin Medical University. The rabbit liver VX2 tumor (Guang Zhou Jennio Biotech Co., Ltd) model was used in this study. Adult male New Zealand 
White rabbits, weighing 2.0-2.5 kg (provided by Harbin Medical University) were anesthetized with an intramuscular injection of $50 \mathrm{mg} / \mathrm{kg}$ zolazepam (Zoletil; Virbac, Carros, France). After a midline subxyphoid abdominal incision, and crushing of VX2 tumor into pieces, approximately $1 \mathrm{~mm}^{3}$ of tumor was directly implanted into the left lobe of liver. The VX2 tumor was allowed to grow for two weeks. The largest tumor, which measured in average $1.5 \mathrm{~cm}$ in diameter by baseline T2-weighted axial MRI, was selected to establish the ALPPS model. The tumor-bearing rabbits were anesthetized by intramuscular injection of $50 \mathrm{mg} / \mathrm{kg}$ zolazepam and maintained on a mixture of $1-3 \%$ isoflurane (RWD Life Science Co, $\mathrm{Ltd})$ and 50\% oxygen and air (0.8-1.5 L/min). The animals were fixed in the Trendelenburg position on the operating table. After a midline laparotomy, the left branch of portal vein was dissected, marked, and ligated with 4-0 silk (Ethicon, Somerville, $\mathrm{NJ}$ ). The ischemic line between the left medial lobe (LML) and the right liver $(\mathrm{RL})$ then became prominent, and the liver parenchyma was then split along the ischemic line [16]. All operations were performed under an operating microscope (Binocular Operation Microscope; Type XTS-4A; Jiangsu Zhenjiangzhongtian Optical Instruments Co, Ltd. China) (Fig.2A).

\section{The ALPPS model in rabbits with liver VX2 tumor}

This study was approved by the Animal Care Committee of Harbin Medical University. The rabbit liver VX2 tumor (Guang Zhou Jennio Biotech Co., Ltd) model was used in this study. Adult male New Zealand White rabbits, weighing 2.0-2.5 kg (provided by Harbin Medical University) were anesthetized with an intramuscular injection of $50 \mathrm{mg} / \mathrm{kg}$ zolazepam (Zoletil; Virbac, Carros, France). After a midline subxyphoid abdominal incision, and crushing of VX2 tumor into pieces, approximately $1 \mathrm{~mm}^{3}$ of tumor was directly implanted into the left lobe of liver. The VX2 tumor was allowed to grow for two weeks. The largest tumor, which measured in average $1.5 \mathrm{~cm}$ in diameter by baseline T2-weighted axial MRI, was selected to establish the ALPPS model. The tumor-bearing rabbits were anesthetized by intramuscular injection of $50 \mathrm{mg} / \mathrm{kg}$ zolazepam and maintained on a mixture of $1-3 \%$ isoflurane (RWD Life Science Co, $\mathrm{Ltd})$ and $50 \%$ oxygen and air $(0.8-1.5 \mathrm{~L} / \mathrm{min})$. The animals were fixed in the Trendelenburg position on the operating table. After a midline laparotomy, the left branch of portal vein was dissected, marked, and ligated with 4-0 silk (Ethicon, Somerville, $\mathrm{NJ}$ ). The ischemic line between the left medial lobe ( $L M L)$ and the right liver $(\mathrm{RL})$ then became prominent, and the liver parenchyma was then split along the ischemic line [16]. All operations were performed under an operating microscope (Binocular Operation Microscope; Type XTS-4A; Jiangsu Zhenjiangzhongtian Optical Instruments Co, Ltd. China).

\section{Synthesis of ${ }^{18} \mathrm{~F}-\mathrm{FDG},{ }^{18} \mathrm{~F}-\mathrm{FLT}$ and ${ }^{18} \mathrm{~F}-\mathrm{FCH}$}

${ }^{18} \mathrm{~F}-\mathrm{FDG}$ was acquired from daily productions at the TOF-PET/CT/MR center of the Fourth Hospital of Harbin Medical University. ${ }^{18} \mathrm{~F}-\mathrm{FLT}$ and ${ }^{18} \mathrm{~F}-\mathrm{FCH}$ were synthesized using the commercially available TRACERlab FX $\mathrm{FN}_{\mathrm{FN}}$ synthesis module (GE Healthcare). ${ }^{18} \mathrm{~F}$-fluorothymidine was synthesized following published methods[17]. In brief, 3-N-Boc-1-[5-0-(4,4'dimethoxytrityl)-3-0-nosyl-2-deoxy-3-D-lyxofuranosyl]thymine( Jiangsu huayi technology Co., Ltd) was used as a precursor and subjected to radiofluorination. ${ }^{18} \mathrm{~F}-\mathrm{FCH}$ was synthesized using the method reported by Melissa E.et al. Ditosylmethane( jiangsu huayi technology Co., Ltd) was fluorinated with fluorine-18 and the 
fluorotosylme-thane was alkylated intermediate with dimethylethanolamine( jiangsu huayi technology Co., Ltd) [18]. The final labeled products of both ${ }^{18} \mathrm{~F}-\mathrm{FLT}$ and ${ }^{18} \mathrm{~F}-\mathrm{FCH}$ had $>95 \%$ radiochemical purity as assessed by high-performance liquid chromatography.

\section{$\mathrm{PET} / \mathrm{CT}$ and MR imaging protocol}

PET/CT imaging was performed on the tumor-bearing rabbits on days $0,1,3,7$, and 14 after surgery. All animals were fasted for at least $6 \mathrm{~h}$ before ${ }^{18} \mathrm{~F}-\mathrm{FDG}$ PET/CT imaging. ${ }^{18} \mathrm{~F}-\mathrm{FDG},{ }^{18} \mathrm{~F}-\mathrm{FLT}$ or ${ }^{18} \mathrm{~F}-\mathrm{FCH}$ (37 MBq/kg) was injected into each rabbit through the marginal ear vein at one separate day individually; PET/CT (GE Discovery PET/CT Elite, American) was performed 50-60 min after tracer injection. The rabbits were fixed in the supine position after anesthesia with intramuscular injection of zolazepam. The CT was performed before the PET. The CT parameters were: $120 \mathrm{kV}, 10 \mathrm{~mA}$, and a $3.33 \mathrm{~mm}$ pitch. Emission data were acquired at $3 \mathrm{~min}$ per bed position. The images were reconstructed using an aniterative reconstruction algorithm to obtain CT, PET, and PET/CT fusion images.

MRI examinations were performed on a 3.0T MR system (GE Discovery MR750W American). After anesthetized, the rabbits were placed in a prone position within the eight channel knee coil (3T HD T/R Knee Array, GE Healthcare). DCE-MRI was performed using a three-dimensional T1-weighted fatsuppressed fast spoiled gradient-echo (3D-FS-FSPGR): TR/TE of 7.7/2.1 ms; FOV of 170×170 mm; imaging acquisition matrix of $272 \times 160$, flip angle of $20^{\circ}$, Slice thickness of $2.5 \mathrm{~mm}$,gap of $1.0 \mathrm{~mm}$. In total, 60 phases were acquired. An intravenous bolus of gadodiamide (GE Healthcare AS) at a dose of $0.1 \mathrm{mmol} / \mathrm{kg}$ was administered by manual injection through the marginal ear vein at $4 \mathrm{ml} / \mathrm{s}$ and was immediately followed by a $5 \mathrm{ml}$ saline flush after the first 12 phases. For T1 mapping, four pre-contrast images were acquired with the same imaging parameters by using different flip angles $\left(5^{\circ}, 10^{\circ}, 20^{\circ}\right.$, and $30^{\circ}$ ). It took approximately $10-11 \mathrm{~min}$ to complete a DCE-MRI sequence with 60 phases, with each phase taking 9s. The total acquisition time of each examination was approximately $15 \mathrm{~min}$.

DCE-MRI data were translated into a quantitative software (Omni-Kinetics, GE Healthcare). The results in the liver were calculated based on dual-input extended Tofts mode[19]. The input function was drawn on the abdominal aorta and the portal vein. The ROI was placed manually in the largest slice of FLR, which outlined the liver shape, excluding the major vessel. A single-input Tofts model was used to fit the tumor[20]. The ROls were used to delineate the entire tumor and avoid the necrosis area. The enhancedtumor on all tumor-containing image slices were selected. The $K^{\text {trans }}$ (volume transfer constant) measures the efflux of the contrast agent from the intravascular space to the extravascular extracellular space (EES). Based these kinetic models, the $\mathrm{K}^{\text {trans }}$ of tumor and FLR were calculated.

\section{Liver volume and volume increase rate measured by MR imaging}

FLR volume (FLRV $\mathrm{MRI}_{\text {I }}$ ) was calculated on a workstation (AW5.0; GE Medical Systems). On $\mathrm{T}_{2} \mathrm{Wl}$, the FLR area was manually delineated with a freehand ROI on all FLR containing images. The total FLRV $\mathrm{MRI}_{\text {Was }}$ calculated using the following equation: 
$\mathrm{FLRV}_{\mathrm{MRI}}=\Sigma \mathrm{FLR}$ area on each FLR-containing slicex(Slice thickness + Gap)

The percentage FLR volume increase after ALPPS was based on the FLRV $V_{\text {MRI }}$ data and calculated using the formula.

$\mathrm{FLRV}_{\text {MRI }}$ volume increase rate $=\frac{\left(\mathrm{FLRV}_{\text {MRI post-ALPSs-FLRV }} \text { MRI pre-ALPPS }\right)}{\text { FLRV }_{\text {MRI pre-ALPS }}} \times 100 \%$

\section{PET/CT data analysis}

For the result of ${ }^{18} \mathrm{~F}-\mathrm{FDG},{ }^{18} \mathrm{~F}-\mathrm{FLT}$ and the ${ }^{18} \mathrm{~F}-\mathrm{FCH}$, the region of interest (ROI) was drawn on the tumor and the FLR area; The maximum standardized uptake value (SUV $\mathrm{Sax}_{\text {max }}$ ) was calculated by measuring the maximal concentration of radioactivity in a ROI and correcting it for body weight and the injected dose.

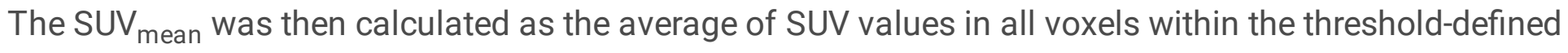
tumor volume. The tumor was automatically delineated on the ${ }^{18} \mathrm{~F}-\mathrm{FDG}$ PET/CT fusion images on workstation (AW5.0; GE Medical Systems) by an outline extraction method in three-dimensional mode (with a threshold of $42 \%$ of the $S U V_{\text {max }}$ ) and then the metabolic tumor volume (MTV) was calculated. The FLR functional volume $\left(\mathrm{FLRV}_{\mathrm{FCH}}\right)$ was manually outlined on the three-dimensional reconstruction of ${ }^{18} \mathrm{~F}$ FCH PET images on a workstation (AW5.0; GE Medical Systems) to exclude adjacent non-liver structures and a threshold of $42 \%$ of the SUV $\max$ within the volumetric region of interest was used to calculate the $\mathrm{FLRV}_{\mathrm{FCH}}$.

\section{Valid volumetric function}

The functional liver is discernible as the region of apparent ${ }^{18} \mathrm{~F}-\mathrm{FCH}$ uptake. To represent the functional liver region (FLR), a Valid Volumetric Function (VVF) was defined as follows:

Valid Volumetric Function (VVF) $=\mathrm{FLR} \mathrm{SUV}_{\text {mean }} \times \mathrm{FLRV}_{\mathrm{FCH}}$

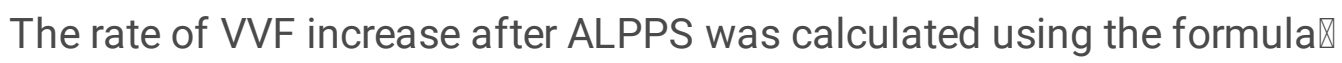

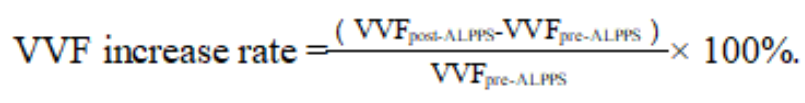

\section{Liver volume measurement by water displacement method}

Three rabbits were selected to be sacrificed. The liver was fully dissected and placed into a bottle filled with water. Liver volume $\left(\mathrm{FLRV}_{\text {water }}\right)$ was equal to the final water volume (with liver) subtracted by the baseline water volume (without liver).

\section{Histopathologic analysis}


After euthanasia, the liver and tumor tissues were fixed in $10 \%$ buffered formalin, embedded, and sectioned for future histopathological analysis. Hepatocyte and tumor cell proliferation was measured by immunohistochemistry with Ki67 antibody (ab15580, Abcam, UK). The sections were also stained with the CD31 antibody (ab199012, Abcam, UK) to measure the angiogenesis and stained with a-SMA (ab7817, Abcam, UK) to access vessel maturation. The microvessel density $₫$ MVD \marked with CD31 was quantified by using the Weidner method as follows: three areas of highest MVD were selected for scoring under high magnification ( $\times 200)$ within each slide, and then the average value of these three areas was recorded as the MVD[21]. Any brown-staining endothelial cell or endothelial cell cluster was considered as a single countable microvessel. The percentage of a-SMA positive stained area was determined by analysing three randomly selected fields at high magnification ( $\times 200)$ from each tumor and FLR section using ImageJ (NIH, Bethesda, MD, USA). The glucose transporter 1 (ab128033, Abcam, UK) was stained to reflect the glucose metabolism. The Ki67-positive rate was quantified with Image $\mathrm{J}$ software (National Institutes of Health, Bethesda, MD). The scores of GLUT1 were estimated according to staining intensity and number of stained cells, as previously described[22]. We also performed hematoxylin and eosin (H\&E) stainings to observe the histopathological.

\section{Statistical analysis}

Data were expressed as mean \pm standard error of the mean (SEM). Differences among each time point and differences between groups were analyzed by two-way analysis of variance. Further data comparison between the two groups at each time point was performed using the variance homogeneity test. The independent or paired t-test was used for equal variance, and the Pearson or Spearman correlation test was used to evaluate correlations between parameters of imaging and histopathologic analyses. All statistical tests were performed with two-tailed distribution and a $P<0.05$ was considered statistically significant. Statistical analyses were performed with GraphPad Prism8.0 (GraphPad Software, San Diego, CA).

\section{Results}

\section{The tumor-bearing rabbit ALPPS model mimicked patients with imaging features}

The first stage of ALPPS was performed in rabbits with VX2 liver tumor under an operating microscope $(n=30)$. Most rabbits survived the procedure and had a smooth recovery. The hepatic artery was the major blood supply of VX2 tumor. Tumor size in the ALPPS group increased more rapidly (from day 14) than in the Sham group $(n=30)$ (Fig. 2B). Although hardly any intercellular substances could be detected by H\&E staining at early time points in the tumor tissues of the two groups, from day 7 onwards more severe necrosis developed in the tumors in the ALPPS group (fig. S1A). FLR hypertrophy became prominent in the ALPPS group from day 7 onwards, but not much changes were detected in the Sham group. H\&E staining of the liver tissue architecture revealed some degree of balloon degeneration, edema, steatosis, and congestion in the FLR of the ALPPS group from day 3 onwards (fig.S1B). 
When ${ }^{18} \mathrm{~F}-\mathrm{FDG},{ }^{18} \mathrm{~F}-\mathrm{FCH}$, and ${ }^{18} \mathrm{~F}-\mathrm{FLT}$ in the same VX2 tumor-carrying rabbit were compared using day 7 as reference (Fig. 2C), ${ }^{18}$ F-FDG PET/CT successfully delineated tumors (with an average SUV $\mathrm{V}_{\text {max }}$ of $6.67 \pm$ $1.05)$ and necrosis areas. However, very low radioactive concentration was detected in the tumors by ${ }^{18} \mathrm{~F}$ FCH and by ${ }^{18} \mathrm{~F}$-FLT PET/CT imagings (Table 1 and fig.S2A). In addition, ${ }^{18} \mathrm{~F}$-FCH successfully delineated the whole liver at all time points in both the ALPPS and Sham groups. ${ }^{18} \mathrm{~F}$-FDG or ${ }^{18} \mathrm{~F}$-FLT showed visually appreciable low contrast in the liver, which was almost identical to the contrast obtained in the muscle (fig. S2 B and C). On ${ }^{18} \mathrm{~F}$-FLT PET imaging, a higher uptake was observed in the bone marrow of the VX2 tumor rabbit model (Fig. $2 \mathrm{C}$ and fig.S2C), which was consistent with the results in previous reports on animal models and humans.

Table 1

$\mathrm{SUV}_{\max }$ of ${ }^{18} \mathrm{~F}-\mathrm{FDG},{ }^{18} \mathrm{~F}-\mathrm{FCH},{ }^{18} \mathrm{~F}-\mathrm{FLT}$ in tumor and FLR in tumor-bearing rabbit on day 7.

\begin{tabular}{|lllllll|}
\hline \multicolumn{3}{|c}{${ }^{18} \mathrm{~F}-\mathrm{FDG}$} & \multicolumn{3}{c|}{${ }^{18} \mathrm{~F}-\mathrm{FCH}$} & \multicolumn{3}{c|}{${ }^{18} \mathrm{~F}-\mathrm{FLT}$} \\
\\
\hline & tumor & FLR & tumor & FLR & tumor & FLR \\
\hline SUV $_{\max }$ & $6.67 \pm 1.05$ & $1.83 \pm 0.27$ & $2.24 \pm 0.24$ & $4.83 \pm 1.05$ & $0.95 \pm 0.22$ & $0.09 \pm 0.03$ \\
\hline
\end{tabular}

${ }^{18}$ F-FDG PET/CT revealed increased glucose metabolism and rapid tumor growth

The ${ }^{18} \mathrm{~F}-\mathrm{FDG}$ tumor uptake increased in the ALPPS group from day 3 onwards and was significantly higher than in the Sham group on day 7 . The uptake remained relatively stable thereafter (Fig. 3A). There

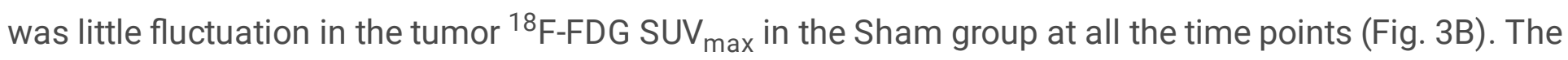
${ }^{18} \mathrm{~F}_{-F D G}$ SUV $\mathrm{max}_{\text {max }}$ in FLR did not change significantly in either the ALPPS or Sham group (fig.S3A and Table 2). The metabolic tumor volume (MTV) as measured by ${ }^{18} \mathrm{~F}-\mathrm{FDG}$ PET/CT increased more rapidly and became higher in the ALPPS group than in the Sham group on day 3, 7, and 14. The difference only became significant on day 14 (Fig. $3 \mathrm{C}$ and Table 2). To further investigate the mechanisms of altered tumor ${ }^{18} \mathrm{~F}-\mathrm{FDG}$ uptake in the ALPPS group, the GLUT1 expression was evaluated by histological examination. Tumors in the ALPPS group had a higher glucose metabolism rate when compared with the Sham group, reaching a peak on day 7 (Fig. 3D and fig. S3B). The GLUT1-positive staining in the tumors correlated positively with ${ }^{18} \mathrm{~F}$-FDG SUV $\max$ (fig.S3C). However, the GLUT1 expression levels in the FLR did not change significantly in either of the two groups (Fig. 3E). 
Table 2

Data of ${ }^{18} \mathrm{~F}-\mathrm{FDG}$ in the ALPPS and Sham groups.

\begin{tabular}{|lllllll|}
\hline Group & MTV $\left(\mathbf{c m}^{3}\right)$ & \multicolumn{3}{c}{ tumor SUV $_{\max }$} & \multicolumn{3}{l|}{ FLR SUV $_{\max }$} \\
\hline Time(d) & ALPPS & Sham & ALPPS & Sham & ALPPS & Sham \\
\hline 0 & $1.42 \pm 0.30$ & $1.39 \pm 0.16$ & $5.99 \pm 1.55$ & $5.96 \pm 2.39$ & $1.97 \pm 0.27$ & $1.86 \pm 0.18$ \\
\hline 1 & $2.09 \pm 0.36$ & $1.73 \pm 0.26$ & $5.55 \pm 2.29$ & $5.20 \pm 0.95$ & $2.01 \pm 0.58$ & $1.71 \pm 0.32$ \\
\hline 3 & $3.85 \pm 1.21$ & $2.48 \pm 0.52$ & $7.32 \pm 2.21$ & $6.54 \pm 1.85$ & $1.99 \pm 072$ & $1.90 \pm 0.50$ \\
7 & $7.19 \pm 2.07$ & $4.62 \pm 1.50$ & $9.99 \pm 1.65^{\star}$ & $6.67 \pm 1.04$ & $2.01 \pm 0.58$ & $1.83 \pm 0.27$ \\
14 & $21.23 \pm 3.05^{\star}$ & $11.97 \pm 2.41$ & $8.62 \pm 2.68$ & $7.26 \pm 1.27$ & $2.05 \pm 0.47$ & $1.87 \pm 0.40$ \\
\hline$* P<0.05 . P<0.05$ was regarded as statistically significant. & & \\
\hline
\end{tabular}

${ }^{18} \mathrm{~F}-\mathrm{FCH}$ detected proliferative function associated with choline metabolically active hepatocytes

Although ${ }^{18} \mathrm{~F}-\mathrm{FCH}$ PET/CT imagings fail to detect the tumors(fig.S3D), they successed in liver(Fig. 4A and Table 3).As the liver is a central organ for choline metabolism, the region of apparent uptake of ${ }^{18} \mathrm{~F}-\mathrm{FCH}$ should reflect metabolically active hepatocytes. To assess the feasibility of using ${ }^{18} \mathrm{~F}$-FCH PET as a noninvasive method to visualize and quantify FLR, the SUV max and $S U V_{\text {mean }}$ in this region were calculated. In the ALPPS group, both the ${ }^{18} \mathrm{~F}-\mathrm{FCH} \mathrm{SUV} \mathrm{max}_{\text {max }}$ and $\mathrm{SUV}_{\text {mean }}$ in FLR increased significantly from day 1 onwards, reaching a peak on day 7 , and then began to decrease gradually, although they still remained at high levels on day 14 when compared with the Sham group (Fig. 4A-C). There was no obvious fluctuation in ${ }^{18} \mathrm{~F}-\mathrm{FCH}$ uptake in the FLR of the Sham group at different time points. To further investigate the mechanisms of altered ${ }^{18} \mathrm{~F}-\mathrm{FCH}$ uptake as observed in the ALPPS group, Ki67 immunocytochemistry staining was performed on FLR and tumor histological sections. A significant increase in the number of proliferative hepatocytes was detected in FLR of the ALPPS group from day 1 to day 14, but not in the Sham group (Fig. 4D and E). Moreover, strong correlation was found between the Ki67-positive rate with

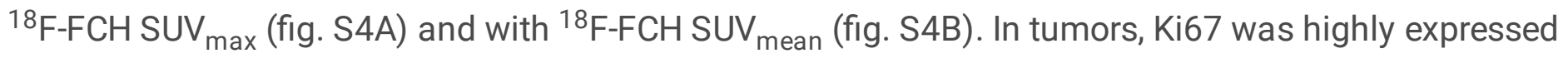
both in the ALPPS and the Sham groups. 
Table 3

Uptake of ${ }^{18} \mathrm{~F}-\mathrm{FCH}$ in the ALPPS and Sham groups.

\begin{tabular}{|lllllll|}
\hline & FLR SUV & \multicolumn{3}{c}{ FLR SUV } \\
\hline Time(d) & ALPPS & Sham & ALPPS & Sham & ALPPS & Sham \\
\hline 0 & $4.62 \pm 0.41$ & $4.70 \pm 0.60$ & $3.50 \pm 0.60$ & $3.49 \pm 1.13$ & $2.25 \pm 0.67$ & $2.20 \pm 0.69$ \\
1 & $6.93 \pm 1.03^{*}$ & $4.80 \pm 0.61$ & $5.25 \pm 0.87^{*}$ & $3.35 \pm 0.65$ & $2.04 \pm 0.66$ & $1.77 \pm 0.71$ \\
3 & $10.27 \pm 1.08^{*}$ & $4.86 \pm 0.79$ & $8.36 \pm 0.37^{*}$ & $3.04 \pm 0.37$ & $1.71 \pm 0.14$ & $1.78 \pm 0.68$ \\
7 & $12.62 \pm 2.60^{*}$ & $4.83 \pm 0.56$ & $9.85 \pm 1.38^{*}$ & $3.02 \pm 0.57$ & $2.85 \pm 0.83$ & $2.24 \pm 0.64$ \\
14 & $7.35 \pm 0.81^{*}$ & $5.22 \pm 0.44$ & $5.20 \pm 0.35^{*}$ & $3.17 \pm 0.39$ & $1.86 \pm 0.13$ & $2.03 \pm 0.67$ \\
\hline$* P<0.05 . P<0.05$ was regarded as statistically significant. & & & \\
\hline
\end{tabular}

\section{${ }^{18}$ F-FCH PET/CT reflected hepatic functional reserve and postoperative liver regeneration}

In the ALPPS group, the functional volume of FLR ( $\left.\mathrm{FLRV}_{\mathrm{FCH}}\right)$ as measured by ${ }^{18} \mathrm{~F}-\mathrm{FCH}$ PET increased from day 1 , and around day 7 and 14 it became significantly different from the Sham group $(P<0.05)$. No significant changes were detected in the $\mathrm{FLRV}_{\mathrm{FCH}}$ of the Sham group at any time point (Fig. 5, A and B, Supplemental video). Strong correlations were found between $\mathrm{FLRV}_{\mathrm{FCH}}$ and $\mathrm{FLRV}_{\text {water }}$ (fig. S4C), and between FLRV $\mathrm{FCH}_{\mathrm{F}}$ and FLRV $\mathrm{MRI}_{\mathrm{MI}}$ (fig.S4D). However, FLRV $\mathrm{MRI}_{\mathrm{MI}}$ was consistently higher than $\mathrm{FLRV}_{\mathrm{FCH}}$ in the ALPPS group (Fig. 5C). As the FLR volume as measured by CT or MR is typically used to identify patients for liver resection, the percentages of FLR volume increase based on MR imaging data were analyzed(Table S1).

The concept of valid volumetric function (VVF, product of mean SUV and FLRV $\mathrm{FCH}_{\text {) }}$ ) is to more accurately reflect the entire functional capacity of FLR. The VVF of FLR in the ALPPS group increased significantly from day 1 , to reach a peak on day 7 , and then decreased gradually to day 14 (Fig. 4D). Using the preoperative VVF as reference, the VVF growth rates on day $1,3,7$, and 14 after ALPPS were $60.42 \pm$ $36.35 \%$ (range $27.39-99.36 \%$ ), $200.83 \pm 82.23 \%$ (126.99-289.44\%), $336.50 \pm 109.47 \%$ (213.21$422.28 \%$ ), and $202.79 \pm 89.67 \%$ (107.77-285.91\%), respectively. However, the VVF of FLR in the Sham group showed no significant changes and remained at the baseline (Table S2).

\section{DCE-MRI detected vascular response in tumor and FLR after ALPPS}

As changes in parameter $\mathrm{K}^{\text {trans }}$ are associated with alterations in hyperpermeable vasculature, DCE-MRI was used to assess vascular responses (Fig. 6.A and B).In the ALPPS group, $\mathrm{K}^{\text {trans }}$ increased suddenly in tumors on day $7(P<0.05)$, and then decreased slightly, but remained higher than the Sham group (Fig. 6C). In contrast, for FLR in the ALPPS group, $\mathrm{K}^{\text {trans }}$ increased gradually, peaked on day 7 and then declined; while in the Sham group, $K^{\text {trans }}$ remained at the baseline level at all time points (Fig. 6D). 
Angiogenesis in tumor and FLR was then assessed with CD31.In the ALPPS group, the CD31 MVD counts in tumor increased rapidly until day $7(P<0.05)$ and then continued to increase more gradually. In the Sham group, CD31 MVD counts in tumor increased slowly at all time points (Fig. 6E). In FLR in the ALPPS group, CD31 MVD counts increased significantly when compared to the Sham group, reaching a peak on day 7 , and remained elevated until day $14(P<0.05)$. For the Sham group, MVD counts maintained at the baseline level at all time points (Fig. 6E). These results strongly correlated with the $\mathrm{K}^{\text {trans }}$ measurements as described above (fig.S6, A and B).

Vessel maturation in tumor and FLR was assessed with a-SMA immunostaining. In the ALPPS group, the a-SMA-positive area (\%) increased rapidly to reach a peak on day 7 in tumor $(P<0.05)$. However, in the Sham group it increased gradually (Fig. 6F). Similarly, in the ALPPS group the a-SMA -positive area (\%) in FLR increased significantly to peak on day 7 , then decreased slowly, but it still remained significantly higher than the Sham group on day $14(P<0.05)$ (Fig. 6F). These results strongly correlated with the $\mathrm{K}^{\text {trans }}$ measurements (fig.S6, C and D).

\section{Discussion}

To conduct ALPPS safely, the first stage of ALPPS should have sufficient function and volume in FLR to prevent PHLF [23], and the second stage of ALPPS should be performed as soon as possible to limit tumor progression [24]. Thus, both the pathophysiological mechanisms of the tumor and FLR should be monitored simultaneously after the first stage of ALPPS to determine the optimal time for the second stage of ALPPS. Over the past decades, technologies for molecular and cellular functional imagings have been increasingly used to elucidate biological processes and pathophysiology. In this study, a comprehensive assessment was conducted on volume, glucose metabolism, choline metabolism, proliferation, and vascular functional changes in tumors and FLR after the first stage of ALPPS in the rabbit liver VX2 tumor model using multiplex molecular and functional imaging methods. The result was validated ex vivo using histological staining.

Previous studies in ALPPS have been conducted on models in mice [25], rats [26],pigs [27], and on an ALPPS model in tumor-free rabbits [16]. However, researches using these models showed only changes in FLR, and hence the influence of ALPPS on tumors remains unclear [25, 27]. In this study, an ALPPS model was established in rabbits with liver VX2 tumor to study the pathophysiological changes in tumor and FLR after the first stage of ALPPS. The results revealed that ALPPS on the tumor-bearing rabbit model showed similar pathological features as in patients, including tumor growth with necrotic regions, and with the hepatic artery being the major blood supply to tumors. Moreover, as longitudinal imaging studies could easily be performed with this model and changes in tumor volume has been used as a standard therapeutic response marker to estimate tumor growth [28], this study showed that the metabolic tumor volume (MTV) was significantly higher in the ALPPS model than in Sham animals, suggesting that ALPPS accelerated tumor growth. These results are consistent with previous data obtained after PVL and PVE $[29,30]$. Importantly, the ${ }^{18} \mathrm{~F}-\mathrm{FDG}$ uptake in tumor revealed that alterations in metabolism preceded volume changes. This finding was confirmed by immunocytochemistry with GLUT1. Furthermore, $\mathrm{K}^{\text {trans }}$ 
DCE-MRI showed that tumor vascular permeability increased significantly from day 7 after ALPPS to reach a high level, and Ki67, CD31 and a-SMA immunostainings showed proliferation and angiogenesis. Thus, in ALPPS, quantitative SUV $\max { }^{18} \mathrm{~F}-\mathrm{FDG}$ PET/CT and $\mathrm{K}^{\text {trans }}$ DCE-MR imagings revealed tumor progression before volume changes became apparent. Also, tumors in the ALPPS group had larger necrotic regions than those in the Sham group after day 7. Moreover, the normal vascular fraction was destroyed, even though neovascularization was still occurring on day 14. On the other hand, in Sham animals these were not seen. Previous studies reported that after blocking portal blood flow to tumors led to increase in arterial blood supply as a compensatory mechanism, with a consequential improvement in tumor nutritional supply which ultimately promoted tumor growth and progression [31]. Although our data supported these changes as tumor progression was detected to accelerate on day 7 in the ALPPS model, it is still difficult to determine which is the cause and which is the consequence, or whether these two processes interact.

While ${ }^{18} \mathrm{~F}$-FLT can be used to monitor therapy responses of tumor in human, in the VX2 tumor ALPPS rabbit model it is unsuitable to use as ${ }^{18} \mathrm{~F}$-FLT PET/CT failed to detect cell proliferation in VX2 tumors and in FLR, despite the high proliferation rates detected in the ALPPS group by Ki67 immunostaining. This observation can be explained by high levels of endogenous thymidine in rabbit serum when compared with human, which would compete with ${ }^{18} \mathrm{~F}-\mathrm{FLT}$ to bind to TK-1 to inhibit ${ }^{18} \mathrm{FFLT}$ uptake [32]. Another possible explanation is that with increased glycosidic phosphorylation, human liver has a higher uptake of ${ }^{18} \mathrm{~F}$-FLT which is a compensatory mechanism that other mammals lack [33]. Also, ${ }^{18} \mathrm{~F}-\mathrm{FCH}$ was also found to have a negligible uptake in VX2 tumors in the ALPPS and control animals. The possible explanation is that as VX2 tumors do not have a hepatic origin, they do not have choline metabolism.

The liver is an important organ of choline metabolism and has an enormous proliferative capacity. During cell proliferation, hepatocytes synthesize phosphatidylcholine (PtC), the most abundant membrane phospholipid. As membrane phospholipid synthesis is linked to cell cycle [11, 12], radiolabeled choline can be used to reflect proliferation by highlighting membrane lipid synthesis [34, 35]. Indeed, our data showed that the uptake of ${ }^{18} \mathrm{~F}-\mathrm{FCH}\left(\mathrm{SUV}_{\text {max }}\right.$ and $\left.\mathrm{SUV}_{\text {mean }}\right)$ in FLR significantly increased after the first stage of ALPPS, and a high level of Ki67 expression was detected by immunocytochemistry.The ${ }^{18} \mathrm{~F}-\mathrm{FCH}$ PET technology could measure functional volume $\left(F L R V_{F C H}\right)$, a volume parameter, with better accuracy than FLRV $V_{\text {water }}$ or FLRV $\mathrm{MRI}_{\text {I. }}$. However, the changes in $\mathrm{FLRV}_{\mathrm{FCH}}$ and choline metabolic function in the ALPPS model were not synchronous, as ${ }^{18} \mathrm{~F}-\mathrm{FCH}$ uptake in FLR began to decrease after day 14 , yet $\mathrm{FLRV}_{\mathrm{FCH}}$ continued to rise. For these reasons, a novel evaluation reference, $\mathrm{VVF}$, was introduced to more accurately assess the functional capacity of FLR. By incorporating the parameters of SUV $\mathrm{V}_{\text {mean }}$ and $\mathrm{FLRV}_{\mathrm{FCH}}$ to determine VVF, the level of active choline metabolism within the total volume of the regions of interest was accounted for. Our data showed that the VVF of FLR in the ALPPS group increased significantly from day 1 , to reached a peak on day 7 and then decreased from day 14 , while the FLRV $F C H$ remained elevated. Despite our attempts to assess glucometabolic function in FLR with ${ }^{18} \mathrm{~F}$-FDG PET/CT imaging, a low level of GLUT1 expression in liver cells resulted in a continuous and low ${ }^{18}$ F-FDG SUV $\max$ 
uptake in both the ALPPS and control animals. Thus, the pathophysiological mechanisms after the first stage of ALPPS do not include accelerated glucometabolism relatated with GLUT1 in liver cells [36, 37].

Quantitative DCE-MRI showed that vascular permeability in FLR increased rapidly after portal vein ligation, which then decreased. These changes correlated well with changes in VVF in the ALPPS model. Combining the results obtained by histological staining in FLR in ALPPS with Ki67 and angiogenesis immunostaining, the vascular functional changes could be interpreted as neovascularization, as there were increase in MVD counts and reduced pericyte coverage of surviving vessels. Neovascularization is required in postoperative liver regeneration to meet the increased needs of hepatic functional reserve [38, 39]. Importantly, our results revealed a reduction in hepatic functional reserve and vascular maturation after day 7 , suggesting there is a critical time window for liver regeneration. A recent study suggests that partial transection of at least $50 \%$ of liver parenchyma could induce a liver hypertrophy level comparable to those obtained with complete transection (137\% vs $156 \%$ ), the latter being the standard procedure for the first stage of ALPPS [40]. As VVF takes into consideration both the volumetric and hepatic functional reserve factors, ${ }^{18} \mathrm{~F}$-FCH VVF should allow an accurate monitoring of dynamics and time window of functional liver regeneration of FLR. In the ALPPS model, the VVF growth rate reached approximately $200 \%$ on day 3 and $336 \%$ on day 7 , with no significant differences between these time points. Notably, tumor pathophysiologic processes were found also to accelerate as early as on day 7 . Thus, taken together, our data strongly suggests that between days 3 to 7 is the optimal time for the second stage of ALPPS to be carried out in the ALPPS model.

The pathophysiological mechanisms after the first stage of ALPPS are complex, as they are influenced by many factors including acceleration of tumor growth and progression, liver hypertrophy, and functional liver regeneration. Our study combined the molecular and functional imaging technologies to uncover the pathophysiological mechanisms of tumor and FLR in ALPPS, and to uncover the optimal time window for the second stage of ALPPS in the rabbit liver VX2 tumor model. In addition, ${ }^{18} \mathrm{~F}-\mathrm{FCH}$ PET/CT using the quantitative VVF parameter was shown to be a promising and non-invasive imaging tool to evaluate hepatocyte proliferation and functional reserve in patients undergoing ALPPS. There are some limitations of this study. The technical challenges to establish an animal model coupled with the need for imagings at various specific time points limited the number of animals in the experimental group. Moreover, the data obtained in animals might not be able to extrapolate to human. Thus, translational research of these results into clinical studies are needed to further investigate the pathophysiological mechanisms of ALPPS in human.

\section{Conclusion}

In summary, ${ }^{18} \mathrm{~F}-\mathrm{FCH}$ PET provided a highly accurate assessment of liver morphology and regeneration and revealed the optimal time for second stage of ALPPS in rabbit models, which should be between 3 to 7 days. ${ }^{18}$ F-FDG PET/CT and $\mathrm{K}^{\text {trans }}$ DCE-MR imaging detect tumor progression after first stage operation in ALPPS earlier than volume changes. The molecular and functional imaging is a suitable non-invasive 
method to accurately visualize and quantify liver regeneration and to assess the influence of surgery on tumor growth and progression. This study will help guide clinical treatment of liver cancer for surgery.

\section{Abbreviations}

ALPPS, associating liver partition and portal vein ligation for staged hepatectomy; CT, Computed tomography; ${ }^{18} \mathrm{~F}$-FDG, ${ }^{18} \mathrm{~F}$-fluorodeoxyglucose; ${ }^{18} \mathrm{~F}$-FLT, 3 -deoxy-3'- ${ }^{18} \mathrm{~F}$-fluorothymidine; ${ }^{18} \mathrm{~F}-\mathrm{FCH},{ }^{18} \mathrm{~F}$ methylcholine; FLR, Future liver remnant; PET, Positron emission tomography; ROI, region of interest; SUV, Standard uptake value; GLUT1, glucose transporter 1;a-SMA,a-Smooth muscle actin; PHLF, posthepatectomy liver failure; PVL, portal vein ligation ; PVE, portal vein embolization; DCE-MRI, Dynamic contrast-enhanced magnetic resonance imaging; IHC, immunohistochemistry; H\&E, Hematoxylin and eosin; MVD, microvessel density

\section{Declarations}

Ethics approval and consent to participate[This study was approved by the Animal Care Committee of Harbin Medical University

Consent for publication $\square$ Not applicable

Availability of data and materials: Not applicable

Competing interests: The authors declare that they have no conflict of interest.

Funding: This study was supported by grants from the National Natural Science Foundation of China (81627901, 81471724) and National Basic Research Program of China (2015CB931800), Heilongjiang Province Foundation for Returned Overseas Chinese Scholars, and the Key Laboratory of Molecular Imaging Foundation (College of Heilongjiang Province), Natural Science Foundation of Heilongjiang Province. (General Program, H2017024).

Acknowledgements: Not applicable

\section{Authors' contributions:}

RFW and ZQ are joint first authors. XLS and RFW obtained funding and designed the study. ZQ, TSZ, KW, $Y L, Z G H, X C W$ and SLM collected the data. RFW and ZQ were involved in data cleaning, and verification. RFW and ZQ analyzed the data. ZQ drafted the manuscript. XLS, WYL and LXL contributed to the interpretation of the results and critical revision of the manuscript for important intellectual content and approved the final version of the manuscript. All authors have read and approved the final manuscript. XLS and RFW are the study guarantors.

\section{References}


1. Ferlay J, Soerjomataram I, Dikshit R, Eser S, Mathers C, Rebelo M, Parkin DM, Forman D, Bray F. Cancer incidence and mortality worldwide: sources, methods and major patterns in GLOBOCAN 2012. Int J Cancer. 2015;136:E359-86.

2. Fong ZV, Tanabe KK. The clinical management of hepatocellular carcinoma in the United States, Europe, and Asia: a comprehensive and evidence-based comparison and review. Cancer. 2014;120:2824-38.

3. Kishi Y, Abdalla EK, Chun YS, Zorzi D, Madoff DC, Wallace MJ, Curley SA, Vauthey JN. Three hundred and one consecutive extended right hepatectomies: evaluation of outcome based on systematic liver volumetry. Ann Surg. 2009;250:540-8.

4. Lau WY, Lai EC, Lau SH. Associating liver partition and portal vein ligation for staged hepatectomy: the current role and development. Hepatobiliary Pancreat Dis Int. 2017;16:17-26.

5. Schnitzbauer AA, Lang SA, Goessmann H, Nadalin S, Baumgart J, Farkas SA, Fichtner-Feigl S, Lorf T, Goralcyk A, Hörbelt R, et al. Right portal vein ligation combined with in situ splitting induces rapid left lateral liver lobe hypertrophy enabling 2-staged extended right hepatic resection in small-for-size settings. Ann Surg. 2012;255:405-14.

6. Ch T, Croome KP, Sergeant G, Cano V, Schadde E, Ardiles V, Slankamenac K, Clariá RS, de Santibaňes E, Hernandez-Alejandro R, et al. Salvage parenchymal liver transection for patients with insufficient volume increase after portal vein occlusion - an extension of the ALPPS approach. Eur J Surg Oncol. 2013;39:1230-5.

7. de Santibañes E, Ardiles V, Alvarez FA. Associating Liver Partition and Portal Vein Ligation for Staged Hepatectomy: A Better Approach to Treat Patients With Extensive Liver Disease. JAMA Surg. 2015;150:929-30.

8. Rahnemai-Azar AA, Cloyd JM, Weber SM, Dillhoff M, Schmidt C, Winslow ER, Pawlik TM. Update on Liver Failure Following Hepatic Resection: Strategies for Prediction and Avoidance of Post-operative Liver Insufficiency. J Clin Transl Hepatol. 2018;6:97-104.

9. Kim JH, Kim CK, Park BK, Park SY, Huh SJ, Kim B. Dynamic contrast-enhanced 3-T MR imaging in cervical cancer before and after concurrent chemoradiotherapy. Eur Radiol. 2012;22:2533-9.

10. Leach MO, Morgan B, Tofts PS, Buckley DL, Huang W, Horsfield MA, Chenevert TL, Collins DJ, Jackson A, Lomas D, et al. Imaging vascular function for early stage clinical trials using dynamic contrast-enhanced magnetic resonance imaging. Eur Radiol. 2012;22:1451-64.

11. Michel V, Yuan Z, Ramsubir S, Bakovic M. Choline transport for phospholipid synthesis. Exp Biol Med (Maywood). 2006;231:490-504.

12. Bansal A, Shuyan W, Hara T, Harris RA, Degrado TR. Biodisposition and metabolism of [(18)F]fluorocholine in 9L glioma cells and 9L glioma-bearing fisher rats. Eur J Nucl Med Mol Imaging. 2008;35:1192-203.

13. Beyoğlu D, Idle JR. The metabolomic window into hepatobiliary disease. J Hepatol. 2013;59:842-58.

14. Bieze M, Klümpen HJ, Verheij J, Beuers U, Phoa SS, van Gulik TM, Bennink RJ. Diagnostic accuracy of (18) F-methylcholine positron emission tomography/computed tomography for intra- and 
extrahepatic hepatocellular carcinoma. Hepatology. 2014;59:996-1006.

15. Chalaye J, Costentin CE, Luciani A, Amaddeo G, Ganne-Carrié N, Baranes L, Allaire M, Calderaro J, Azoulay D, Nahon P, et al. Positron emission tomography/computed tomography with $18 \mathrm{~F}$ fluorocholine improve tumor staging and treatment allocation in patients with hepatocellular carcinoma. J Hepatol. 2018;69:336-44.

16. Liao M, Zhang T, Wang H, Liu Y, Lu M, Huang J, Zeng Y. Rabbit model provides new insights in liver regeneration after transection with portal vein ligation. J Surg Res. 2017;209:242-51.

17. Lee SJ, Oh SJ, Chi DY, Kil HS, Kim EN, Ryu JS, Moon DH. Simple and highly efficient synthesis of 3'deoxy-3'-[18F]fluorothymidine using nucleophilic fluorination catalyzed by protic solvent. Eur J Nucl Med Mol Imaging. 2007;34:1406-9.

18. Rodnick ME, Brooks AF, Hockley BG, Henderson BD, Scott PJ. A fully-automated one-pot synthesis of [18F]fluoromethylcholine with reduced dimethylaminoethanol contamination via [18F]fluoromethyl tosylate. Appl Radiat Isot. 2013;78:26-32.

19. Li Z, Sun J, Chen L, Huang N, Hu P, Hu X, Han G, Zhou Y, Bai W, Niu T, et al. Assessment of liver fibrosis using pharmacokinetic parameters of dynamic contrast-enhanced magnetic resonance imaging. J Magn Reson Imaging. 2016;44:98-104.

20. Joo I, Lee JM, Grimm R, Han JK, Choi BI. Monitoring Vascular Disrupting Therapy in a Rabbit Liver Tumor Model: Relationship between Tumor Perfusion Parameters at IVIM Diffusion-weighted MR Imaging and Those at Dynamic Contrast-enhanced MR Imaging. Radiology. 2016;278:104-13.

21. Weidner N. Current pathologic methods for measuring intratumoral microvessel density within breast carcinoma and other solid tumors. Breast Cancer Res Treat. 1995;36:169-80.

22. Roh MS, Jeong JS, Kim YH, Kim MC, Hong SH. Diagnostic utility of GLUT1 in the differential diagnosis of liver carcinomas. Hepatogastroenterology. 2004;51:1315-8.

23. Pawlik TM, Schulick RD, Choti MA. Expanding criteria for resectability of colorectal liver metastases. Oncologist. 2008;13:51-64.

24. Rohatgi S, Harrison EM, Powell JJ, Wigmore SJ. ALPPS: Adverse Outcomes Demand Clear Justification in an Era of Improving Survival for Colorectal Liver Metastases. World J Surg. 2015;39:1848-9.

25. Schlegel A, Lesurtel M, Melloul E, Limani P, Tschuor C, Graf R, Humar B, Clavien PA. ALPPS: from human to mice highlighting accelerated and novel mechanisms of liver regeneration. Ann Surg. 2014;260:839 - 46; discussion 846-7..

26. Wei W, Zhang T, Zafarnia S, Schenk A, Xie C, Kan C, Dirsch O, Settmacher U, Dahmen U. Establishment of a rat model: Associating liver partition with portal vein ligation for staged hepatectomy. Surgery. 2016;159:1299-307.

27. Croome KP, Mao SA, Glorioso JM, Krishna M, Nyberg SL, Nagorney DM. Characterization of a porcine model for associating liver partition and portal vein ligation for a staged hepatectomy. HPB (Oxford). 2015;17:1130-6. 
28. Braren R, Altomonte J, Settles M, Neff F, Esposito I, Ebert O, Schwaiger M, Rummeny E, Steingoetter A. Validation of preclinical multiparametric imaging for prediction of necrosis in hepatocellular carcinoma after embolization. J Hepatol. 2011;55:1034-40.

29. Sakai N, Clarke CN, Schuster R, Blanchard J, Tevar AD, Edwards MJ, Lentsch AB. Portal vein ligation accelerates tumor growth in ligated, but not contralateral lobes. World J Gastroenterol. 2010;16:3816-26.

30. Fülöp A, Szijártó A, Harsányi L, Budai A, Pekli D, Korsós D, Horváth I, Kovács N, Karlinger K, Máthé D, et al. Demonstration of metabolic and cellular effects of portal vein ligation using multi-modal PET/MRI measurements in healthy rat liver. PLoS One. 2014;9:e90760.

31. Gock M, Eipel C, Linnebacher M, Klar E, Vollmar B. Impact of portal branch ligation on tissue regeneration, microcirculatory response and microarchitecture in portal blood-deprived and undeprived liver tissue. Microvasc Res. 2011;81:274-80.

32. Schelhaas S, Heinzmann K, Bollineni VR, Kramer GM, Liu Y, Waterton JC, Aboagye EO, Shields AF, Soloviev D, Jacobs AH. Preclinical Applications of 3'-Deoxy-3'-[(18)F]Fluorothymidine in Oncology - A Systematic Review. Theranostics. 2017;7:40-50.

33. Shields AF, Grierson JR, Dohmen BM, Machulla HJ, Stayanoff JC, Lawhorn-Crews JM, Obradovich JE, Muzik O, Mangner TJ. Imaging proliferation in vivo with [F-18]FLT and positron emission tomography. Nat Med. 1998;4:1334-6.

34. Yoshimoto M, Waki A, Obata A, Furukawa T, Yonekura Y, Fujibayashi Y. Radiolabeled choline as a proliferation marker: comparison with radiolabeled acetate. Nucl Med Biol. 2004;31:859-65.

35. Kuang Y, Salem N, Tian H, Kolthammer JA, Corn DJ, Wu C, Wang F, Wang Y, Lee Z. Imaging lipid synthesis in hepatocellular carcinoma with [methyl-11c]choline: correlation with in vivo metabolic studies. J Nucl Med. 2011;52:98-106.

36. Seatter MJ, Gould GW. The mammalian facilitative glucose transporter (GLUT) family. Pharm Biotechnol. 1999;12:201-28.

37. Joost HG, Thorens B. The extended GLUT-family of sugar/polyol transport facilitators: nomenclature, sequence characteristics, and potential function of its novel members (review). Mol Membr Biol. 2001;18:247-56.

38. Bertens KA, Hawel J, Lung K, Buac S, Pineda-Solis K, Hernandez-Alejandro R. ALPPS: challenging the concept of unresectability-a systematic review. Int J Surg. 2015;13:280-7.

39. de Santibañes M, Dietrich A, Alvarez FA, Ardiles V, Loresi M, D'adamo M, de Santibañes E. Biological Substrate of the Rapid Volumetric Changes Observed in the Human Liver During the Associating Liver Partition and Portal Vein Ligation for Staged Hepatectomy Approach. J Gastrointest Surg. 2016;20:546-53.

40. Linecker M, Kambakamba P, Reiner CS, Linh Nguyen-Kim TD, Stavrou GA, Jenner RM, Oldhafer KJ, Björnsson B, Schlegel A, Györi G, et al. How much liver needs to be transected in ALPPS? A translational study investigating the concept of less invasiveness. Surgery. 2017;161:453-64. 


\section{Figures}

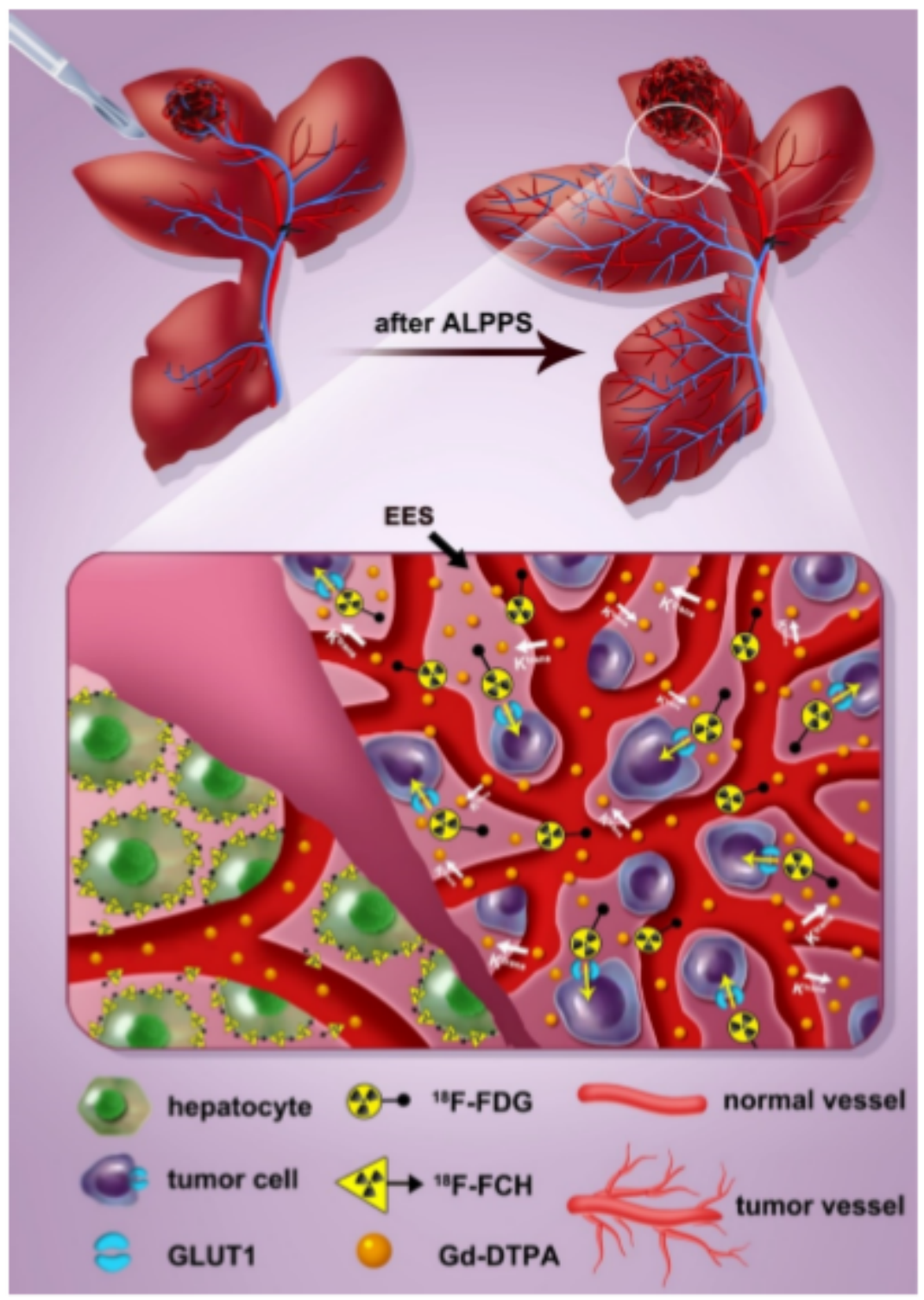

\section{Figure 1}

Schematic representation of the physiopathologic changes after ALPPS. The mechanisms of uptake for multiplexed PET tracers included 18F-FDG in liver tumor cells, 18FFCH in normal hepatocytes, and DCE compartments of vascular bed, cells, and EES (GLUT1 = glucose transporter-1; EES= extravascular extracellular space). 
A
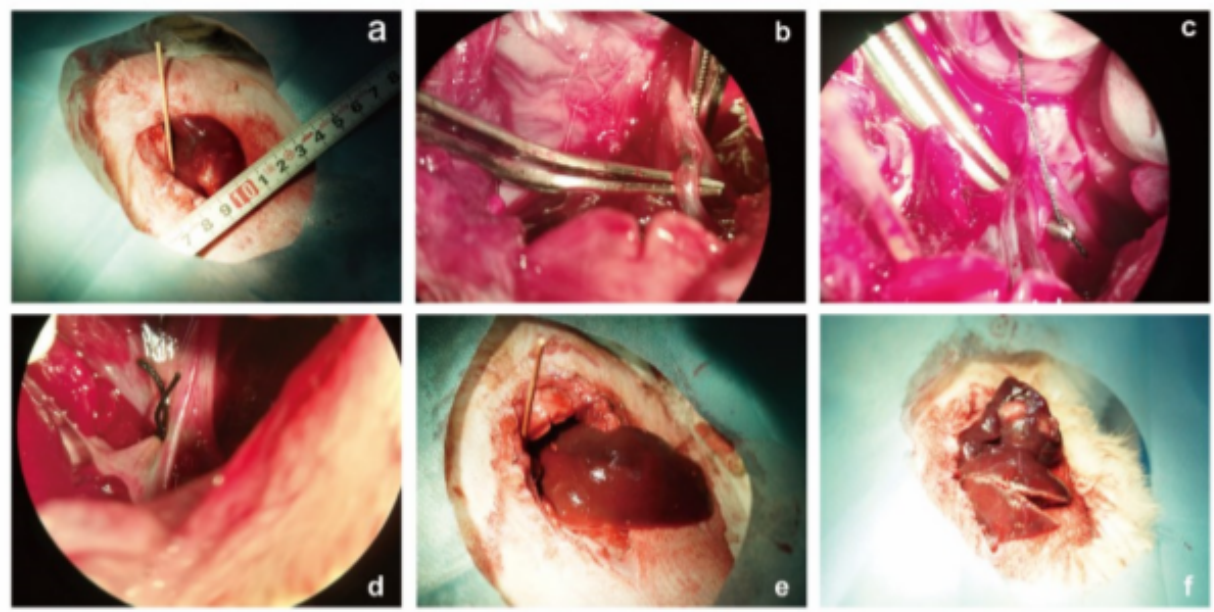

B

Time after ALPPS(day)

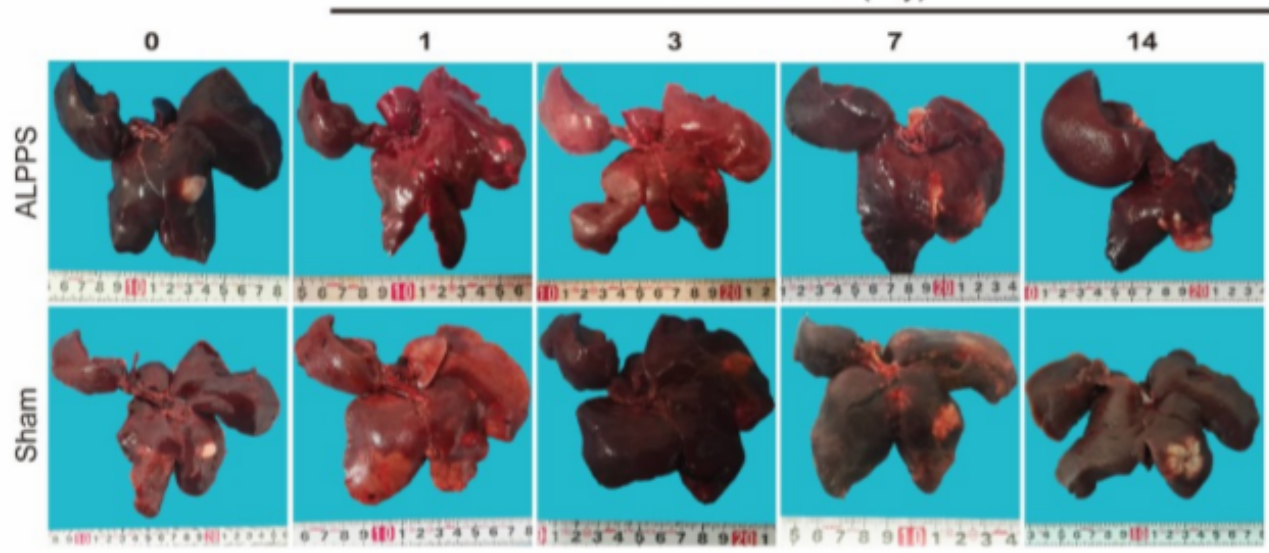

C

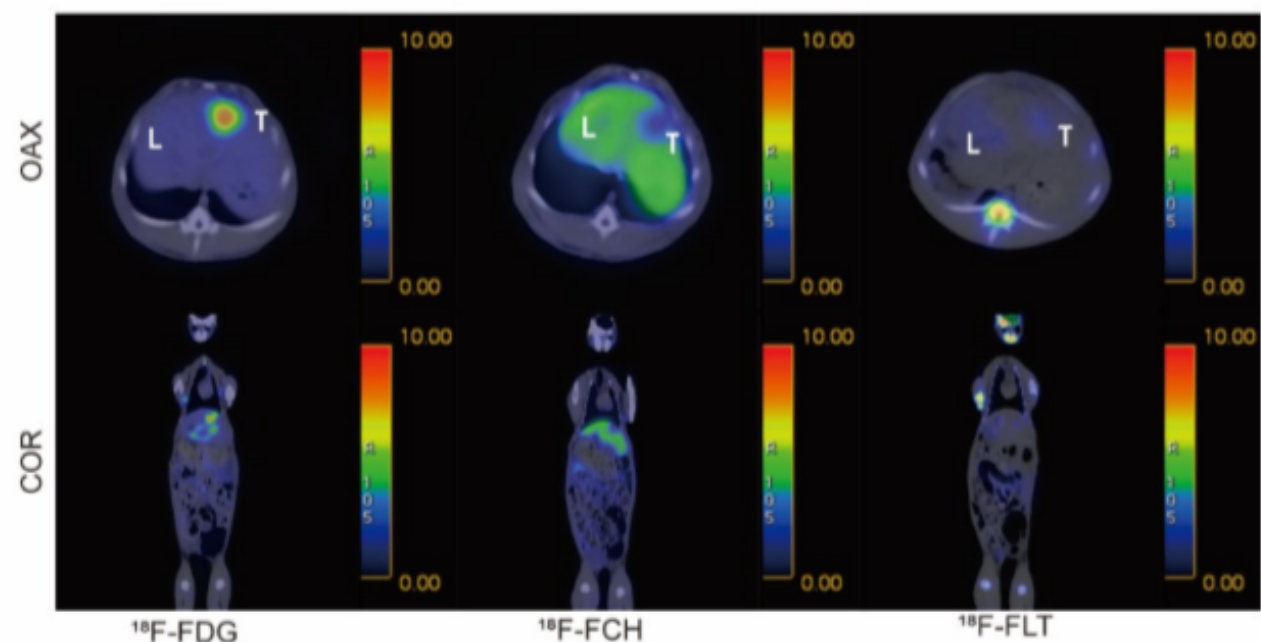

\section{Figure 2}

The first stage of ALPPS in the model of VX2 tumor-bearing rabbits and PET/CT imaging with multiplexed radiotracers. (A) Main surgical procedures for liver parenchyma transection and portal vein branch ligation in the model of rabbit with VX2 tumor. (a) Rabbit with a tumor diameter up to $1.5 \mathrm{~cm}$, was chosen for ALPPS. (b) The Glisson sheath was found under surgical microscopy. (c) The Glisson sheath was dissected carefully to find left branch of portal vein. (d) Ligation of left branch of portal vein. (e) 
Ischemic line became prominent. (f) Liver parenchyma was split from the free edge to the anterior wall of inferior vena cava along the ischemic line. (B) The sizes of liver and tumor of the ALPPS group gradually increased, compared with the Sham group at different time points. (C) 18F-FDG, 18FFCH, 18F-FLT $\mathrm{PET} / \mathrm{CT}$ in a VX2 tumor-bearing rabbit. T: tumor and L: liver.

A

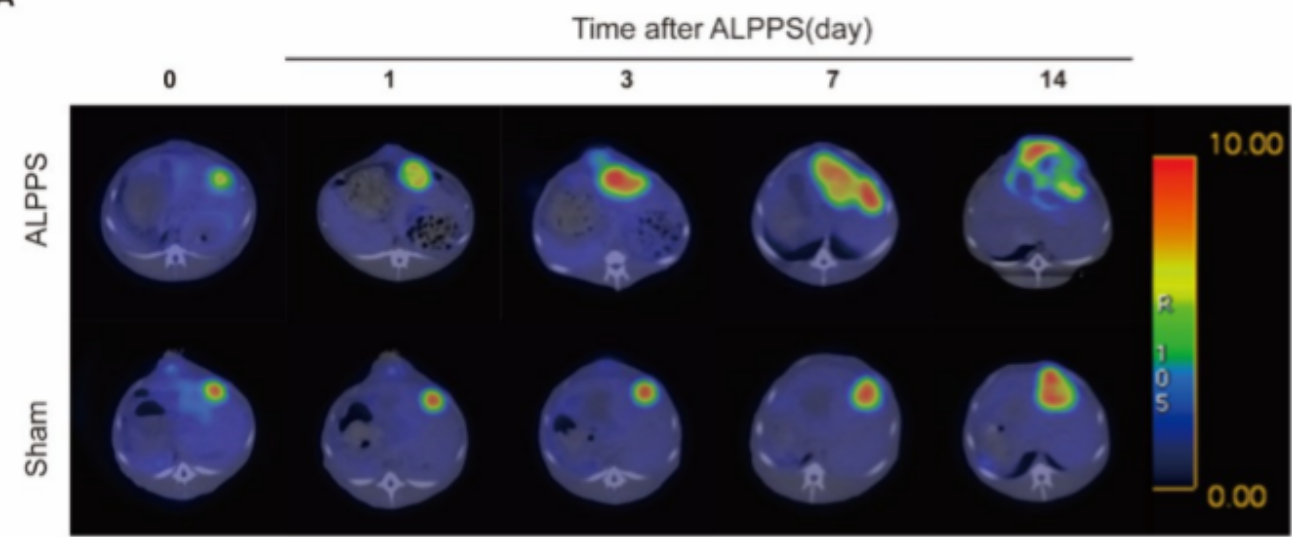

B
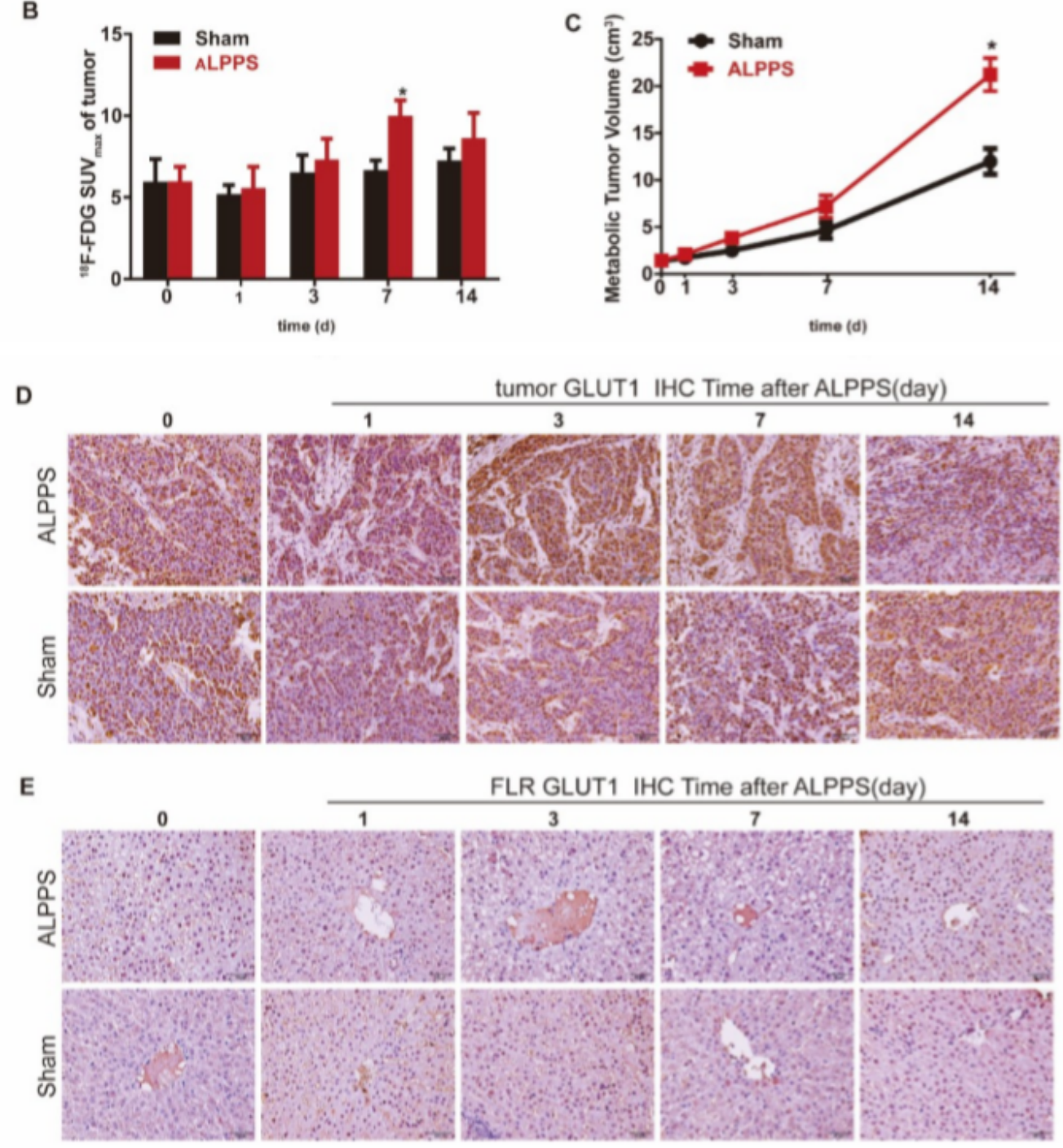

Figure 3 
18F-FDG PET/CT in the ALPPS group and Sham groups. (A) 18F-FDG PET/CT fusion imaging at different time points (Axial position). (B) 18F-FDG SUVmax of tumor in the ALPPS groups was significantly higher than the Sham group on day $7\left({ }^{\star} \mathrm{P}<0.05\right)$. (C) The tumor MTV measured by $18 \mathrm{~F}-\mathrm{FDG}$ PET/CT was higher in the ALPPS group than the Sham group on day 3,7 , and 14 , the difference was only statistically significant on day $14\left({ }^{\star} \mathrm{P}<0.05\right)$. (D) Immunocytochemistry (IHC) staining of tumor GLUT1 expression in the two groups (original magnification $\times 200$ ). (E) IHC staining of FLR GLUT1 expression in the two groups (original magnification $\times 200$ ).

A

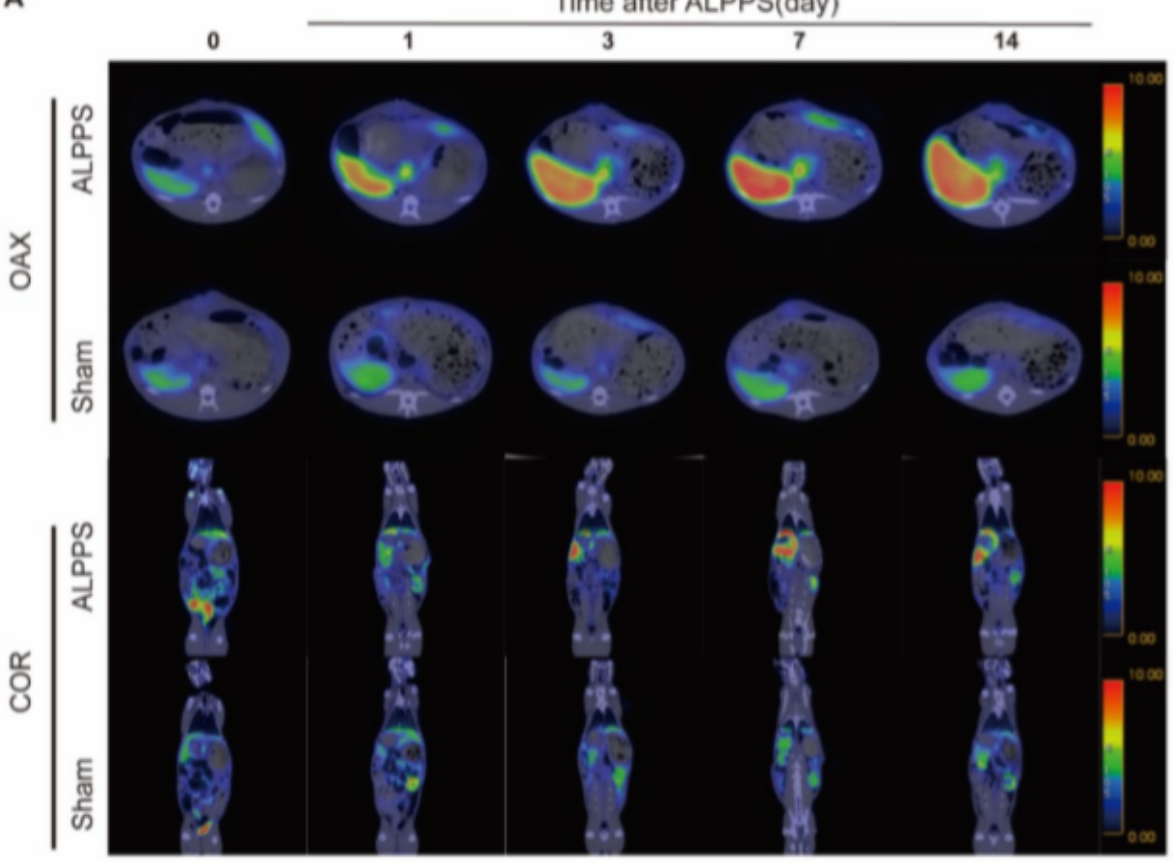

B

C

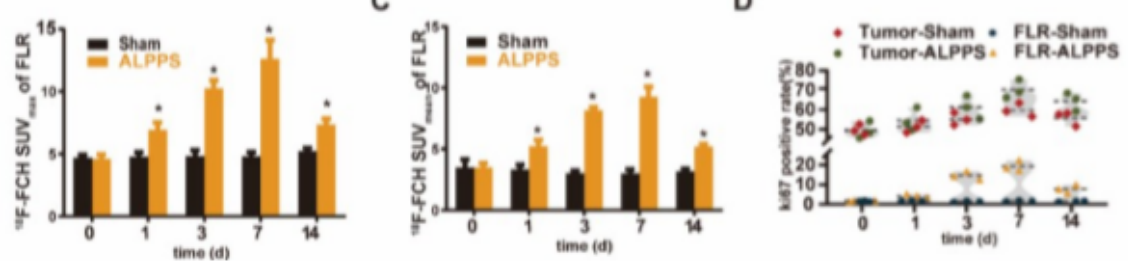

E

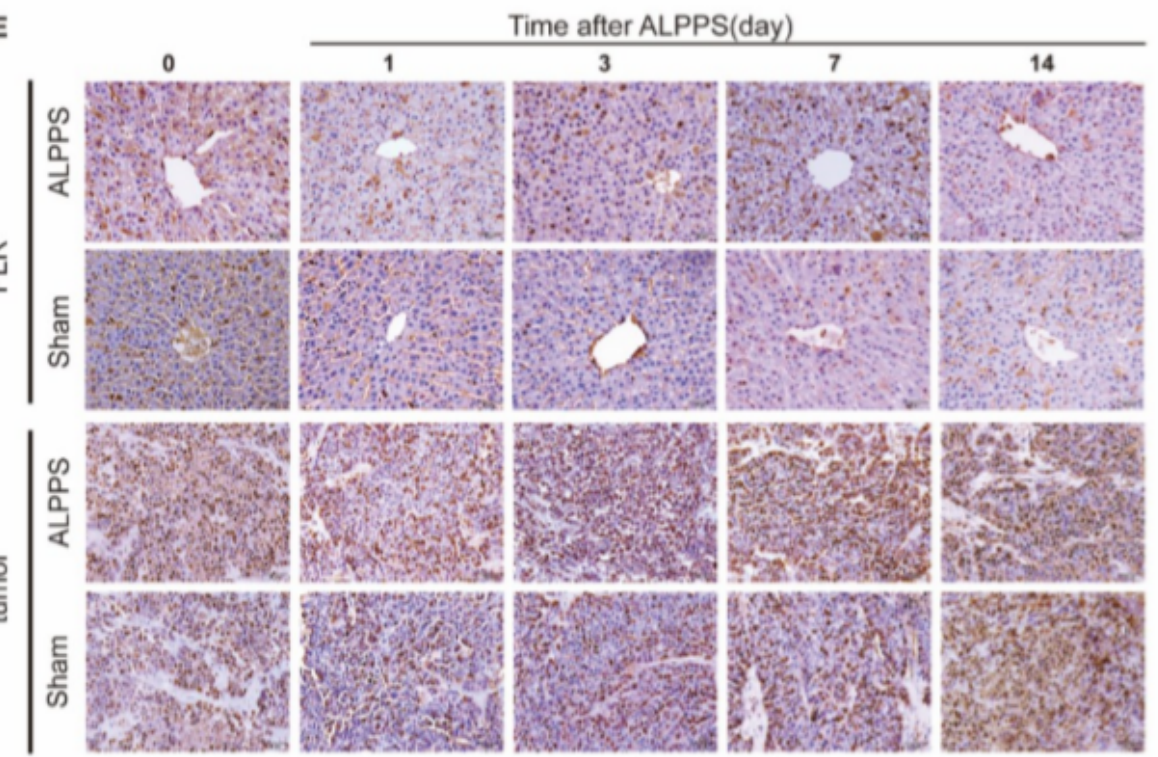




\section{Figure 4}

18F-FCH PET/CT in both ALPPS and Sham groups. (A) 18F-FCH PET/CT fusion imaging at different time points (Axial and Coronal position). (B) 18F-FCH SUVmax of FLR increased in the ALPPS group compared to the Sham group from day 1 . The peak was on day 7 , and began to decrease gradually, the level on day 14 compared with the Sham group ( $\left.{ }^{*} \mathrm{P}<0.05\right)$. (C) $18 \mathrm{~F}-\mathrm{FCH}$ SUVmean of FLR also increased in the ALPPS group compared to the Sham group from day 1 The peak was on day 7 ( $\left.{ }^{\star} P<0.05\right)$. (D) Ki67positive rate of tumor and FLR in the two groups using a violin plot at different time points. (E) IHC staining of proliferation in the tumor and FLR by Ki67 staining in the ALPPS and Sham group (original magnification $\times 200$ )

A

Time after ALPPS(day)

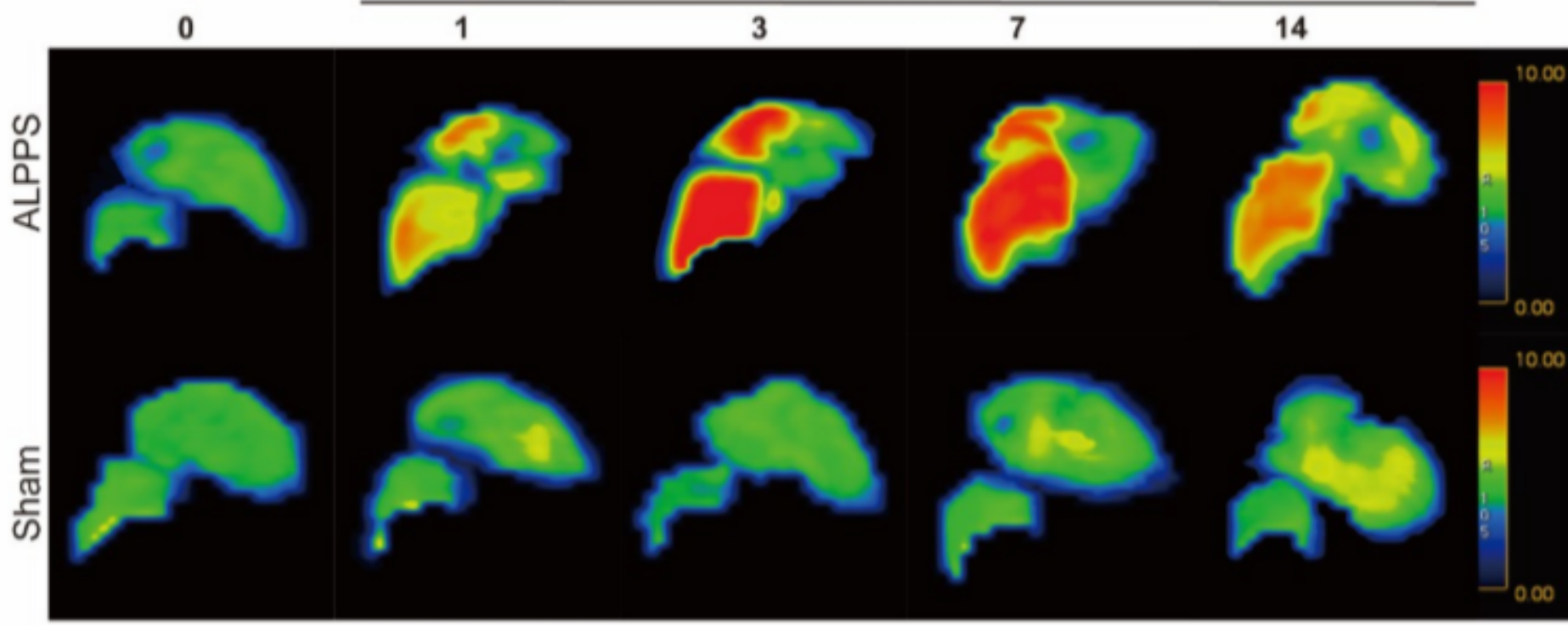

B
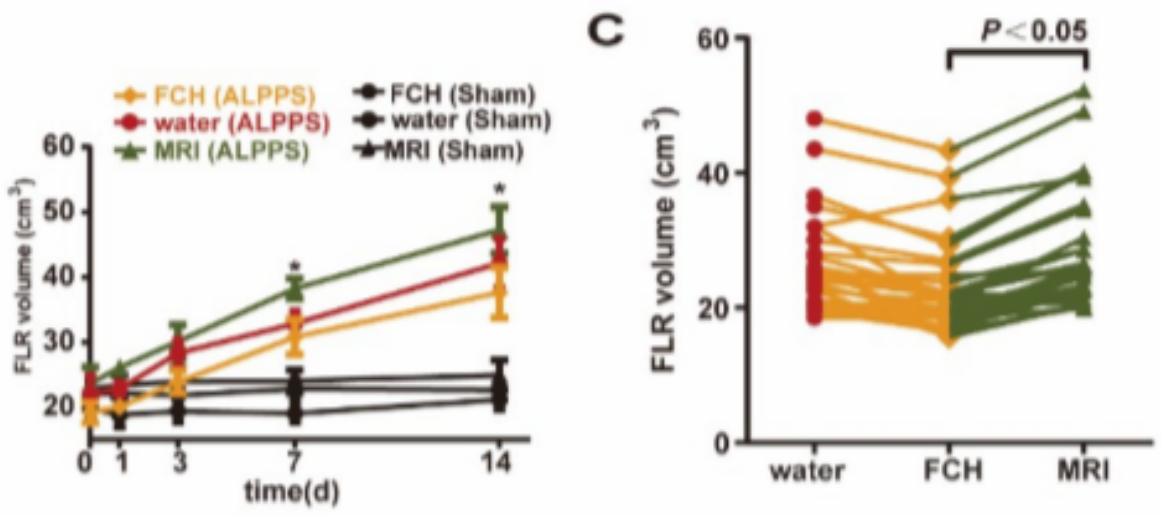

D

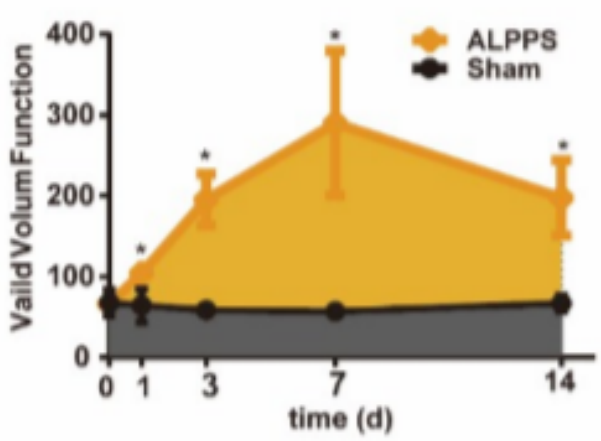

\section{Figure 5}

Functional volume of FLR was measured by $18 \mathrm{~F}-\mathrm{FCH}$ PET/CT in the ALPPS and Sham groups. (A) 18FFCH PET imaging at different time points (Three-dimensional reconstruction of the liver). (B) FLR volume measured by $18 \mathrm{~F}-\mathrm{FCH}$ PET, water displacement method and MRI, the differences were statistically significant on day 7 and $14\left({ }^{*} \mathrm{P}<0.05\right)$. (C) The differences between FLRVFCH and FLRVMRI were 
statistically significant $\left({ }^{*} \mathrm{P}<0.05\right)$. (D) The valid volumetric function (VVF) used as a reference to reflect the actual liver proliferation capacity at different time points $\left({ }^{*} \mathrm{P}<0.05\right)$.

A

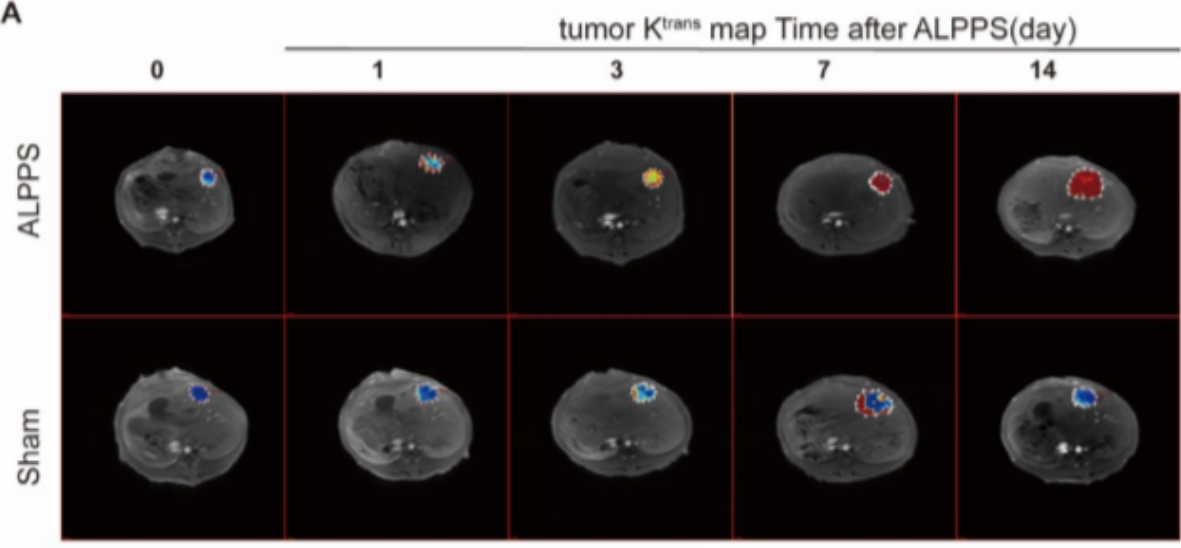

B

$\begin{array}{ccc}\text { FLR K }^{\text {trans }} \text { colormap Time after ALPPS(day) } & \\ 3 & 7 & 14\end{array}$

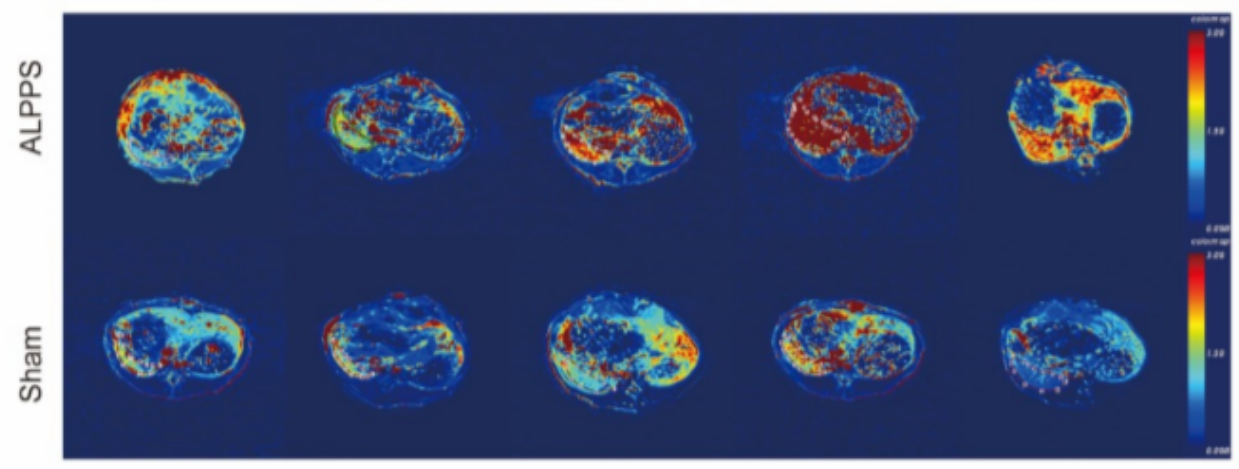

C

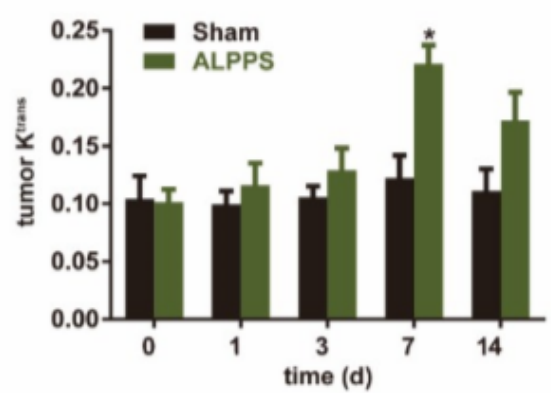

E

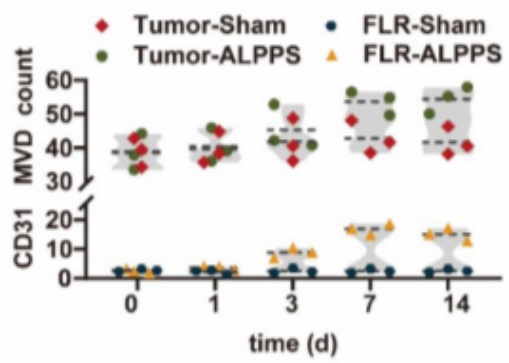

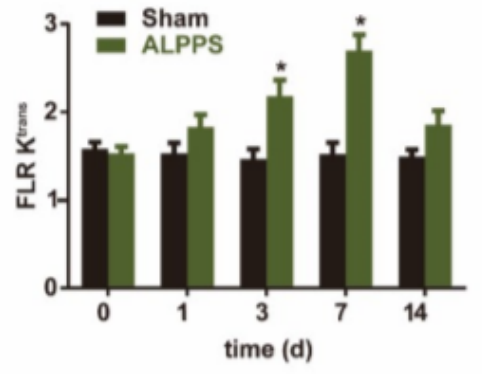

$\mathbf{F}$

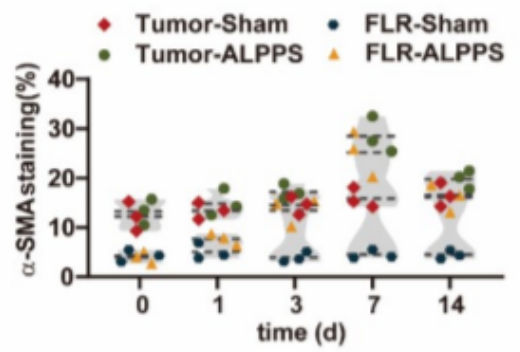

Figure 6

Vascular response of tumor and FLR in the ALPPS and Sham groups were monitored by DCE-MRI. (A) Ktrans map of tumor at different time points based on the Tofts model. (B) Ktrans colormap of FLR at different time points based on the Extend tofts model. (C) Tumor Ktrans measured by DCE-MRI in both 
groups at different time points, the difference was statistically significant on day $7\left({ }^{*} P<0.05\right)$. (D) FLR Ktrans measured by DCE-MRI in both groups at different time points, the differences were statistically significant on day 3 and $7\left({ }^{*} P<0.05\right)$. (E)The angiogenesis assessed by CD31 MVD counts in tumor and FLR in a violin plot of both groups. (F) The vessel maturation assessed by $\mathbb{X}$-SMA-positive stained area (\%) of tumor and FLR in a violin plot of both groups at different time points.

\section{Supplementary Files}

This is a list of supplementary files associated with this preprint. Click to download.

- Supplementalvideo.mp4

- Supplementallnformation.docx 